\title{
The ability of different strains of rats to acquire a visual nonmatching-to-sample task
}

\author{
JOHN P. AGGLETON \\ University of Wales, College of Cardiff, Cardiff, Wales
}

\begin{abstract}
Rats of four different strains (DA, Fischer 344, PVG hooded, and Sprague-Dawley) were trained on a nonspatial recognition task. The task, delayed-nonmatching-to-sample, required the rat to select between two complex stimuli on the basis of their relative familiarity. The four strains differed markedly. The two pigmented strains (DA and PVG) were significantly better than the two albino strains (Fischer 344 and Sprague-Dawley); among the pigmented strains, the DA rats consistently had the higher scores. The Fischer 344 rats had the lowest scores, and their pattern of errors indicated that they were performing in a qualitatively different manner from the other three strains. A control test with the two pigmented strains confirmed that they were able to use visual cues to solve the task. The results point to the likely value of the DA strain for such experiments and highlight potential confounding variables arising from the use of different strains for psychobiological research.
\end{abstract}

A number of new tests of one-trial visual learning and memory have been developed for rats in recent years. The purpose behind their introduction has been the desire to broaden the range of questions that can be addressed with this species. Furthermore, by modeling these tasks on tests given to other animals it is hoped to increase cross-species comparability. One example concerns the development of a number of tests of visual recognition (Aggleton, 1985; Ennaceur \& Delacour, 1988; Mumby, Pinel, \& Wood, 1990; Rothblat $\&$ Hayes, 1987). These have typically been modeled on the delayed-nonmatching-to-sample (DNMS) paradigm that has proved so influential in the study of primate memory (Gaffan, 1974; Mishkin, 1978). The present study compared the ability of four different strains of rat to perform such a task. By testing for differences in the rate of acquisition, the present study sought to identify those strains most suitable for such research and, at the same time, to uncover factors associated with the performance of this task.

The DNMS procedure involves giving a subject a forced choice between two stimuli: one that is novel, and one that is familiar. In the nonmatching task, the animal is rewarded for selecting the novel stimulus. The procedure used in the present study involved running rats in a Y-maze in which the animal is given a choice between two arms: one that contains a goalbox that is the same as that which the animal has just left, and one that is different (i.e., unfamiliar; see Aggleton, 1985). New stimuli are used for each trial within a session, but stimuli are repeated on average every fifth session. Because the location of the correct arm changes in a randomized manner, the task cannot be solved by a spatial strategy. This version of the DNMS task has been used to assess the effects of a range of se-

This research was supported by a grant from the Medical Research Council. Correspondence should be addressed to J. P. Aggleton, School of Psychology, University of Wales, College of Cardiff, P. O. Box 901 , Cardiff CF1 3YG, Wales (e-mail: aggleton@cardiff.ac.uk). lective lesions and drugs (Aggleton, Hunt, \& Rawlins, 1986; Huston \& Aggleton, 1987; Kolb, Buhrmann, McDonald, \& Sutherland, 1994; Rawlins, Lyford, Seferiades, Deacon \& Cassaday, 1993).

Four strains of rat were used in this study: two pigmented and two albino. One of the pigmented strains (DA) was included because it has been used in the majority of previous studies using this task. The other pigmented strain (PVG) was included because it is inbred, to match the DA rats, and is derived from the hooded Lister strain, which is very widely used in psychobiological research. Both of the albino strains, Sprague-Dawley and Fischer 344 , are commonly used in behavioral experiments, the Fischer 344 strain being particularly favored in research into the neurobiology of aging (Bartus \& Dean, 1985).

\section{METHOD}

\section{Subjects}

The subjects were 25 male rats all supplied by Bantin and Kingman (Hull, U.K.). They comprised 6 dark agouti rats (strain DA/ $\mathrm{Bk} 1$ ), 6 hooded rats (strain PVG/Bk1), 6 Fischer 344 rats (strain F344/Bk1), and 7 Sprague-Dawley rats (strain SDG). The first two strains are pigmented; the last two are albino. The DA, PVG, and Fischer 344 rats are from inbred strains; the Sprague-Dawley rats represent an outbred strain. All rats were approximately 4 months old at the start of the experiment. Their weights were as follows: DA, $245 \mathrm{~g}$ (range $=242-249 \mathrm{~g}) ; \mathrm{PVG}, 216 \mathrm{~g}($ range $=210-222 \mathrm{~g})$; Sprague-Dawley, $257 \mathrm{~g}$ (range $=235-275 \mathrm{~g}$ ); Fischer $344,251 \mathrm{~g}$ (range $=244-259 \mathrm{~g}$ ).

Throughout the experimental period, the rats were housed individually under diurnal conditions (14:10-h light:dark). All testing occurred at a regular time, approximately half way through the light period. The animals were tested 5 days a week. Prior to test days, they were fed on a restricted diet (Labsure ERM) so that they did not drop below $85 \%$ of normal body weight. All animals had free access to water.

\footnotetext{
Apparatus

The test apparatus consisted of an aluminum Y-maze, each arm of which was $13 \mathrm{~cm}$ wide and $20 \mathrm{~cm}$ high. Fifty pairs of hardboard
} 
boxes served as both startboxes and goalboxes. These boxes, which were $19 \mathrm{~cm}$ high, $12 \mathrm{~cm}$ wide, and $9 \mathrm{~cm}$ deep, fitted into the end of each arm of the maze, forming a total arm length of $26 \mathrm{~cm}$. The appearance of the boxes constituting each pair was made as similar as possible, but each pair was distinct from every other pair. To achieve this, the walls and floors of the boxes were painted in different colors and patterns, and the floors were lined with a variety of materials, such as sandpaper, wooden strips, metal, Perspex, and cloth. In addition, each pair contained an identical object, such as a plastic cup, a metal bracket, or a wooden block; however, no two pairs contained the same object. The floors of the boxes, which extended toward the center of the maze, began $8 \mathrm{~cm}$ from a Y-shaped aluminum guillotine door at the center of the Y-maze. The back end of each box had been cut away so that food pellets ( $45 \mathrm{mg}$, Campden Instruments, Loughborough) could be dispensed, via metal tubes, to the rear of each box. The Y-maze was illuminated by a fluorescent ceiling light $215 \mathrm{~cm}$ above the apparatus, and the luminant light level in the center of the test room (where the Y-maze was placed) was 285 lux.

\section{Procedure}

Following a standard period of 6 days pretraining, which involved placing the rats in the Y-maze and training them to run for food pellets, the experiment proper began. To start each test session, the rat was placed in an arm with a featureless startbox. The central door was then raised, and the ani:nal was allowed to choose between two arms that contained a matching pair of distinctive goalboxes ( $\mathrm{A} 1$ and $\mathrm{A} 2$ ). The rat was deemed to have made a choice when all four paws had entered an arm, whereupon the guillotine door was lowered. On this first run, the animal was rewarded with three food pellets, whichever box it entered. The animal was confined to this box (A1) for $20 \mathrm{sec}$, during which time the other two test boxes were replaced. The central door was then raised, revealing a familiar box (A2) in one arm and a novel box (B1) in the other. The animal was rewarded with three food pellets if it entered the novel box $\mathrm{Bl}$.

After 20-sec confinement in Box B1, the second trial began. The central door was raised, and the animal chose between the now familiar appearance of Box B2 (negative) and a novel box C1 (positive). Since the startbox was still present, there was little or no retention delay. This sequence was repeated with new pairs of boxes for a total of 10 trials, during which selection of the novel box was always rewarded (Trial 3, C2- vs. D1+; .. Trial 10, J2- vs. K1+). A balanced schedule determined whether the correct response was to the right or left. The sequence of test boxes was varied after every 50 trials so that any particular box occurred, on average, in every fifth session.

If an animal made an incorrect choice, correction trials were run with the same set of goalboxes until the animal selected the novel box. During these correction trials, the goalboxes were rearranged so that entering the positive box required the same body turn as in the test trial. These correction trials were necessary in order to ensure that the animal enter the goalbox that was to become the next startbox.

All rats were tested for a total of 25 sessions ( 250 trials). At the completion of these sessions, the two strains with the highest scores (DA and PVG) were tested for a further 10 sessions. This involved 5 standard sessions, as described above, as well as 5 sessions using modified test stimuli. These two classes of session were given on alternate days. It was decided not to test the Sprague-Dawley or the F344 rats on this more difficult condition because the poor levels of performance shown by these animals meant that any resultant comparisons would be compromised by floor effects.

The modified stimuli consisted of patterns of black, gray, and white printed onto photographic paper and pasted onto the front of different-shaped pieces of hardboard. These were then placed in distinctive arrangements within the test boxes. As before, a matching pair was made for each distinctive design. The purpose of this modification was to provide stimuli that would be visually distinctive but could not be identified using olfactory or surface textural cues. Twenty pairs of modified test boxes were used over the 5 test sessions; therefore, a subset of 20 normal pairs of test stimuli were used for the intervening standard sessions.

\section{RESULTS}

There was no evidence that the four strains differed in the number of pretraining sessions that were required before the experiment proper could begin. Figure 1 shows the mean number of correct scores for each consecutive block of 50 trials ( 5 sessions). The individual scores for the first five blocks of sessions were compared using an analysis of variance (ANOVA) with the between factor (group) and the within factor (session block). This revealed highly significant effects of group $[F(3,21)=$ $20.5, p<.001]$ and session $[F(4,12)=12.5, p<.001]$. There was no group $\times$ session interaction $[F(12,84)=$ 1.20]. Subsequent Newman-Keuls tests showed that the DA group outperformed the PVG group $(p<.05)$ and the two albino groups (both $p s<.01$ ). In addition, the PVG group achieved higher scores than did both the SpragueDawley and F344 rats (both $p$ s <.05), and the SpragueDawley rats performed better than did the F344 animals $(p<.05)$.

Next, the performance of the DA and PVG strains over the last two blocks of 50 trials (standard and modified stimuli) was examined (Figure 1). An ANOVA revealed that there was a group effect $[F(1,10)=5.45, p=.042]$, reflecting the superior performance of the DA animals. There was also a stimulus effect $[F(1,10)=24.4, p=$ $.0006]$, reflecting the ability of the animals to perform the task more accurately with complex three-dimensional stimuli (standard) than with the modified visual stimuli. There was no group $\times$ type of stimulus interaction $(F<1)$.

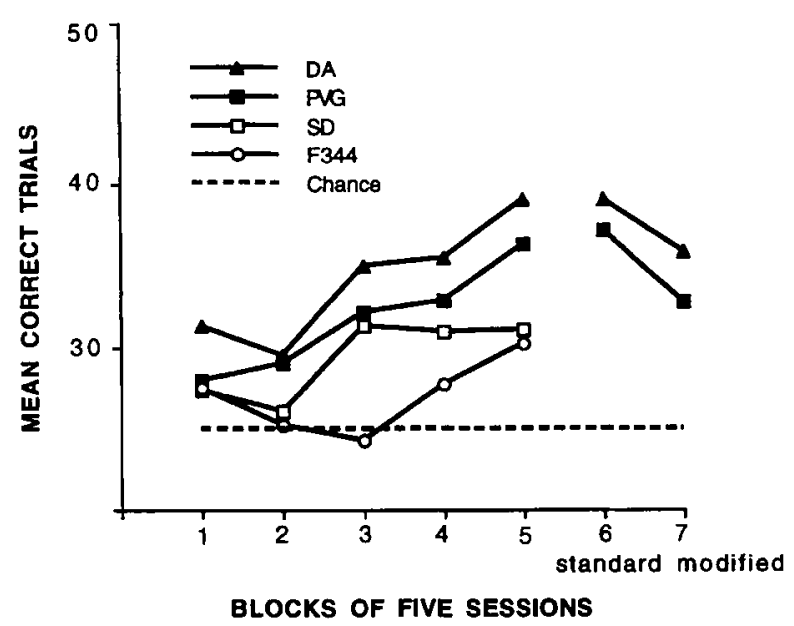

Figure 1. Mean number of correct trials for the four different strains of rat on the delayed-nonmatching-to-sample task. The data have been divided into five blocks, each of 50 trials. Blocks 6 and 7 refer to the control conditions given only to the DA and the PVG strains. 
Analyses using $Z$ scores confirmed that both the DA group and the PVG group performed considerably above chance on the modified condition.

A second series of analyses compared the total number of errors, including those on correction trials, made by the animals from all four groups on the five blocks of standard test sessions (Figure 2). This analysis produced a very clear group effect $[F(3,21)=29.4, p<.001]$, with almost exactly the same pattern of individual group differences as that found for the analysis based on correct scores. The only change was that the total error scores for the F344 and Sprague-Dawley rats did not differ significantly. In addition, there was a highly significant group $\times$ session interaction $[F(12,84)=3.76, p<.001]$, reflecting the abnormal rise in error scores shown by the F344 rats (Figure 2).

This significant interaction prompted a further set of analyses. Previous studies had shown that normal DA rats display an uneven pattern of error scores during the initial stages of acquisition (Shaw \& Aggleton, 1993). This arises because the rats make disproportionately more errors when the correct choice involves returning to the arm that the rat had just come from on the previous trial. That is, they show a bias to avoid the arm visited most recently and so tend to select the different arm. For this reason, the total number of errors made on Trials $2-10$ of the first 10 sessions were calculated and divided between same trials and different trials. A same trial was when the correct response was to return to the just-visited arm. Errors on Trial 1 of each session could not be classified since there was no preceding trial; errors on correction trials were ignored because, in practice, all of these are same trials. This arises because, in a correction trial, the animal is required to make the same body turn as it should have made in the original test trial. This effectively brings the animal back to the location of the previous startbox. The distributions of same- and different-trial errors are shown in Figure 3.

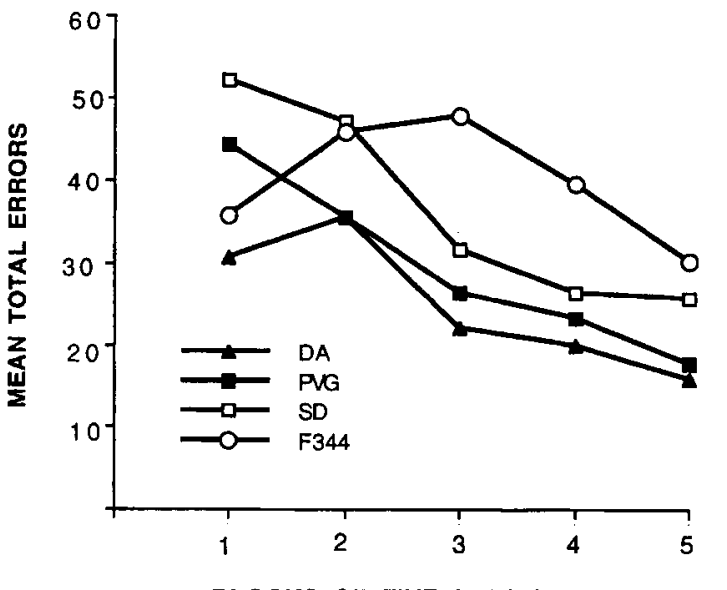

BLOCKS OF FIVE SESSIONS

Figure 2. Mean number of errors made by the four different strains of rat. This includes errors made on correction trials. The data have been divided into five blocks, each of $\mathbf{5 0}$ trials.

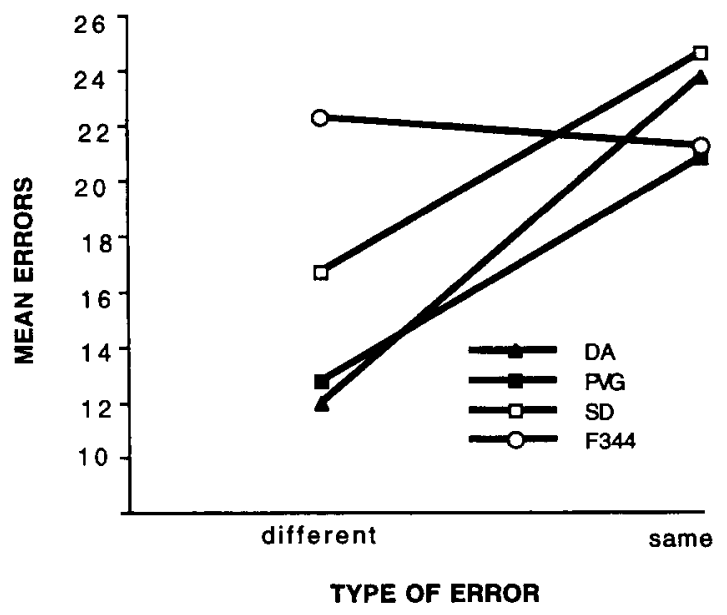

Figure 3. Mean number of errors made over the first 100 trials. Same errors occurred when the correct choice was to return to the same arm from which the animal last came; different errors occurred when the correct choice was the arm that the rat did not last visit. These error scores do not include errors made on correction trials.

An ANOVA revealed significant effects of group $[F(3,20)=5.16, p=.008]$ and type of trial (same or different $)$ on which the error occurred $[F(1,20)=55.8, p<$ $.001]$. The latter effect matched the expectation that more same-trial errors should occur. Of especial interest, however, was the interaction between group and type of trial $[F(3,20)=9.14, p=.005]$. An analysis of the simple effects showed that this was due to the F344 group, which was the only group that failed to show any difference between these two types of trial $(p=.54)$. In contrast, the other three groups all made many more errors on the same trials (all $p \mathrm{~s}<.001$ ). In view of this result, a further comparison examined whether any of the animals had an increased tendency to turn consistently in a given direction (i.e., whether they adopted a fixed body-turn rule during initial learning of this difficult task). As a consequence, the errors made over the first 100 trials were divided between right and left choices, and the ratio of the larger to the smaller number was computed. This included errors made on correction trials. The mean ratios of the four groups were as follows: DA, $1.67(S E=0.62)$; PVG, $1.77(S E=0.25)$; Sprague-Dawley, $1.97(S E=$ $0.37) ; \mathrm{F} 344,1.23(S E=0.07)$. There were no group differences $[F(3,21)=1.34]$.

The abnormal pattern of error scores shown by the F344 group (Figures 2 and 3) led to a further analysis, which compared the mean number of correction trials required once an error had occurred. This was done in order to assess the persistency with which a rat made an incorrect choice. This was computed by dividing the total number of correction trials over the 25 sessions by the number of trials on which correction trials were required. The mean number of correction trials per error were as follows: DA, $1.56(S E=0.043)$; PVG, $1.60(S E=$ $0.055)$; F344, $1.76(S E=0.031)$; Sprague-Dawley, 1.78 $(S E=0.037)$. A one-way ANOVA revealed a clear group effect $[F(3,21)=6.70, p=.0024]$. Subsequent Newman- 
Keuls tests showed that both pigmented strains (DA and PVG) required fewer correction trials per error (all $p \mathrm{~s}<$ .05 ) than did the albino strains (F344 and SpragueDawley). There were no other differences.

\section{DISCUSSION}

The present study revealed a marked difference between the ability of different strains of rat to perform a DNMS task that required the animals to distinguish between familiar and relatively unfamiliar complex stimuli. The results also indicated that the pigmented DA and PVG rats were heavily reliant on visual cues in order to solve the task. Evidence for this comes both from the superior performance of the pigmented strains over the albino strains (Birch \& Jacobs, 1979) and from their DNMS performance on the condition with modified stimuli. In this modified condition, the test stimuli could not be distinguished by olfactory cues or tactile cues because they were all composed of the same material. The superior performance on the standard condition presumably reflects, however, the greater discriminability of the stimuli. Other evidence that this DNMS task taxes visual recognition comes from a previous study using DA rats in which various modifications of test stimuli were assessed (Huston \& Aggleton, 1987).

In contrast to the DA and PVG rats, both albino strains performed poorly on the DNMS task. This presumably reflects the poorer visual acuity of albino rats (Birch \& Jacobs, 1979) and is consistent with the finding that albino rats perform more poorly on a spatial navigation task that taxes the use of distal visual cues (Tonkiss, Shultz, \& Galler, 1992). There were, however, some clear differences between the F344 and Sprague-Dawley strains. The F344 rats performed significantly worse than did the Sprague-Dawley rats and, in fact, were the only strain not to show a marked improvement over the 250 trials. Close inspection of their initial error scores also reveal an unusual pattern of performance. Unlike the three other strains, the F344 rats did not display more errors when the correct choice was to return to an arm from which they had just come. This was not, however, because the strain showed an unusually high bias to turn in a consistent direction. Thus, the choice behavior of the F344 rats seemed considerably more random than that of the other groups. In addition, the apparent rise in total errors made by this group over the first three blocks of sessions (Figure 2) suggests an increasing tendency to perseverate with a choice once an error had been made. This is supported by the finding that the F344 rats required a higher number of correction trials per error than did the pigmented strains.

The Sprague-Dawley rats also required a relatively high number of correction trials, but their performance over the first 100 trials shows that they were sensitive to the locations of the various arms. This combination of error scores suggests that these rats were tending to perseverate in their avoidance of the arm most recently vis- ited. In view of the apparently similar distribution of same and different errors among the DA, PVG, and Sprague-Dawley groups, the issue emerges of why the pigmented strains did not persist for so long in this pattern of behavior, which can be regarded as closely akin to spontaneous alternation. A likely explanation comes from the fact that rats also spontaneously prefer to explore novel objects rather than familiar objects (Aggleton, 1985; Ennaceur \& Delacour, 1988). As a consequence, the nonmatching task accords with the rats' natural tendencies, and this explains why the rats began the task above chance (Figure 1). If, however, the novel objects were more salient to some of the strains (i.e., the pigmented animals), it would explain why they more readily began to drop the inefficient spatial strategy and relied more on the appearance of the novel test boxes to direct their choice behavior.

The present study found that, for this task, the DA rats produced the highest level of nonmatching and were significantly better than the other pigmented strain. Other evidence that the DA strain may be particularly useful for tests of visual learning comes from a recent study that compared the ability of two strains of rats (DA and hooded Lister) to acquire a number of visual discriminations presented on a visual display unit (Gaffan \& Eacott, 1995). As in the present study, the DA strain performed at a higher level, and some of the differences were significant. In view of recent developments concerning the introduction of new automated visual learning tests for rats (Bussey, Muir, \& Robbins, 1994; Gaffan \& Eacott, 1995), these results suggest that the DA strain may be of particular value for future studies. A further benefit of this strain for lengthy behavioral tasks is that DA rats put on relatively little additional weight during adulthood. It is not yet known, however, why the DA strain might be especially proficient at learning such tasks. It does not appear, for example, to be the case that this superiority reflects better visual acuity in the DA strain. A recent study of visual evoked cortical potentials to contrast reversals of sinewave gratings (Heywood, personal communication, June 1995) revealed that DA and hooded Lister rats both have the same acuity (1.1-1.2 cycles per degree). These levels of acuity are, however, far superior to those found in albino rats (Birch \& Jacobs, 1979; Heywood, personal communication, June 1995).

The present study serves to highlight how the choice of strain can impose an additional factor when comparing the outcome of seemingly similar experiments. This was most evident for the F344 strain, which is widely used when testing effects of aging. Not only were these rats very poor on the DNMS task, there was also clear evidence that their pattern of performance was qualitatively different from that of the other rats. Unlike the other strains, they appeared to be insensitive to the spatial locations of the test arms and did not show typical spontaneous biases. This unusual behavior might lead to different ways of solving spatial memory tasks, such as T-maze alternation and the radial-arm maze (e.g., using 
egocentric rather than allocentric cues). This may, in turn, lead to the same neural manipulation having different behavioral outcomes in different strains.

\section{REFERENCES}

AGGLETON, J. P. (1985). One-trial object recognition by rats. Quarterly Journal of Experimental Psychology, 37B, 279-294.

Aggleton, J. P., Hunt, P. R., \& Rawlins, J. N. P. (1986). The effects of hippocampal lesions upon spatial and non-spatial tests of working memory. Behavioral Brain Research, 19, 133-146.

BarTUS, R. T. (1985). Developing and utilizing animal models in the search for an effective treatment for age-related memory disturbances. In C. G. Gottfries (Ed.), Normal aging, Alzheimer's disease and senile dementia (pp. 231-267). Brussels: University of Brussels Press

BIRCH, D., \& JACOBS, G. H. (1979). Spatial contrast sensitivity in albino and pigmented rats. Vision Research, 19, 933-937.

Bussey, T. J., MuIR, J. L., \& RobBins, T. W. (1994). A novel automated touchscreen procedure for assessing learning in the rat using computer graphic stimuli. Neuroscience, 15, 103-110.

ENNACEUR, A., \& DELACOUR, J. (1988). A new one-trial test for neurobiological studies of memory in rats. 1: Behavioural data. Behavioural Brain Research, 31, 47-59.

GAFFAN, D. (1974). Recognition impaired and association intact in the memory of monkeys after transection of the fornix. Journal of Com parative \& Physiological Psychology, 86, 1100-1109.

Gaffan, E. A., \& EacotT, M. J. (1995). A computer-controlled maze environment for testing visual memory in the rat. Journal of Neuroscience Methods, 60, 23-37.
Huston, A. E., \& Aggleton, J. P. (1987). The effects of cholinergic drugs upon recognition memory in rats. Quarterly Journal of Experimental Psychology, 39B, 297-314.

Kolb, B., Buhrmann, K., McDonald, R., \& Sutherland, R. J. (1994). Dissociation of the medial prefrontal, posterior parietal, and posterior temporal cortex for spatial navigation and recognition memory in the rat. Cerebral Cortex, 6, 664-680.

Mishkin, M. (1978). Memory in monkeys severely impaired by combined but not by separate removal of amygdala and hippocampus. Nature, 273, 297-298.

Mumby, D. G., Pinel, J. P. J., \& Wood, E. R. (1990). Nonrecurringitems delayed nonmatching-to-sample in rats: A new paradigm for testing nonspatial working memory. Psychobiology, 18, 321-326.

Rawlins, J. N. P., Lyford, G. L., SEFERIades, A., Deacon, R. M. J., \& CAsSADAY, H. J. (1993). Critical determinants of nonspatial working memory deficits in rats with conventional lesions of the hippocampus or fornix. Behavioral Neuroscience, 107, 420-433.

RothBlat, L. A., \& HAYES, L. L. (1987). Short-term object recognition memory in the rat: Nonmatching with trial-unique junk stimuli. $B e$ havioral Neuroscience, 101, 587-590.

Shaw, C., \& Aggleton, J. P. (1993). The effects of fornix and medial prefrontal lesions on delayed non-matching-to-sample by rats. $B e$ havioural Brain Research, 54, 91-102.

Tonkiss, J., Shultz, P., \& Galler, J. R. (1992). Long-Evans and Sprague-Dawley rats differ in their spatial navigation performance during ontogeny and maturity. Developmental Psychobiology, 25, $567-579$.

(Manuscript received July 26, 1995; revision accepted for publication October 26, 1995. ) 